\title{
ZIPRASIDONE HYDROCHLORIDE LOADED NANOSTRUCTURED LIPID CARRIERS (NLCS) FOR INTRANASAL DELIVERY: OPTIMIZATION AND IN VIVO STUDIES
}

\author{
PRAVEEN SIVADASU ${ }^{1}$, GOWDA D. V. ${ }^{*}$, SIDDARAMAIAH H. ${ }^{2}$, HEMALATHA . $^{3}$
}

${ }^{1}$ Department of Pharmaceutics, JSS College of Pharmacy, JSSAHER, Mysuru 570015, India, ${ }^{2}$ Department of Polymer Science and Technology, Sri Jayachamarajendra College of Engineering, Mysuru 570006, India, ${ }^{3}$ Department of Anaesthesiology, JSS Medical College, JSSAHER, Mysuru 570015, India Email: dvgowda@jssuni.edu.in

\section{Received: 13 Sep 2019, Revised and Accepted: 01 Nov 2019}

\begin{abstract}
Objective: The present study was an attempt to systemically deliver the most desirable schizophrenia drug, ziprasidone hydrochloride (ZRS) via the intranasal route using nanostructured lipid carrier (NLC) approach.
\end{abstract}

Methods: The desired ZRS loaded NLCs were developed using central composite statistical design and the developed formulation was monitored for improving ZRS bioavailability and their brain targeting efficacy.

Results: Pharmacokinetic studies revealed a 10 fold increase (ZRS blood-brain ratio) for NLCs administered through nasal route (in comparison to intravenous route). Similarly, the concentration of ZRS (in the brain) delivered via nasal route exhibits 4 fold increment at all-time points.

Conclusion: Therefore, the obtained results suggest a potential nose to brain transport of loaded ZRS by effective bypassing of the Blood-Brain Barrier (BBB).

Keywords: Ziprasidone hydrochloride, Nanostructured lipid carriers, Blood/Brain barrier, Intranasal delivery

(C) 2020 The Authors. Published by Innovare Academic Sciences Pvt Ltd. This is an open-access article under the CC BY license (http://creativecommons.org/licenses/by/4.0/)

DOI: http://dx.doi.org/10.22159/ijap.2020v12i1.35683 Journal homepage: https://innovareacademics.in/journals/index.php/ijap

\section{INTRODUCTION}

Schizophrenia is considered a chronic health disorder pertaining to mental issues, which can be diagnosed by a range of symptoms like hallucinations, disorientation in both behavior and speech, cognitive impairment and delusive behavior. Further, due to its chronic nature and early-onset schizophrenia is considered as disabling disorder (due to negative and cognitive symptoms) both for patients and their people $[1,2]$. Additionally, due to positive symptoms (hallucinations, delusional behavior and suspiciousness), there is a chance of relapse even after receiving treatment. Finally, due to its characteristic heterogeneousness, there is a lack of understanding regarding various aspects of this disorder [3]. Nevertheless, systemic drug delivery to treat schizophrenia is a potential challenge, owing to Blood-Brain Barrier (BBB) effects, which often obstruct the efficient transport of a rational drug (to the brain) [4].

Techniques employed to deliver drugs to the brain by by-passing bloodbrain-barrier are categorized as invasive and non-invasive methods. Wherein, delivering drugs through invasive route was not always welcomed by the patient since it causes irreversible damage to barrier integrity which leads to severe adverse effects like changes in neuropathological functioning and enhanced contact to toxic molecules. Further, non-invasive delivery of drugs aids direct transport of drugs into the brain by evading surgical intrusions $[5,6]$. In the modern era nose has been considered as a route of delivery for both local and systemic effects. In the last two decades, there are more advances in large scale production of drugs especially proteins and peptides [6].

Nasal cavity possesses a great expanse which affords potential alternative for brain delivery by avoiding first-pass metabolism and invasiveness and swift oncoming, simultaneously increasing the patient ease and compliance. Additionally, the nasal cavity acts as a barrier to transport moieties with high molecular weights like peptides. Further, this hurdle can be overcome by employing permeation enhancers in the formulations which in turn opens up the tight joints of the barrier for paracellular transport of the selected moieties. Nasal delivery also owns other advantages like trouble-free administration and self-medication is possible during critical conditions [7].
Since the beginning of the $20^{\text {th }}$ century, nanotechnology growing interested in pharmaceutical technology research groups worldwide. Further, excipients used in this nanoformulations are generally biocompatible and degradable, so that drugs can be delivered at the required site of action with the controlled release by reducing the toxicity [8]. The method of complete crystallization or recrystallization of fat present in the formulation reduces the solubility of the loaded drug, thereby leading to expulsion of the drug from the formulation especially in lipid nanoparticles. Additionally, most of the lipophilic drugs are highly soluble in a liquid lipid when compared with a solid lipid. Thus, NLC is an updated version for solid lipid nanoparticles (SLN), where both solid and liquid lipids were present in the oil phase are increasingly preferred over SLN, as it overcomes major disadvantages of SLN, such as low drug loading and drug expulsion related issues [9-11].

In the current study, a second-generation, atypical antipsychotic drug Ziprasidone hydrochloride (ZRS) is selected. Further, it has a half-life of $7 \mathrm{~h}$ and requires repeated dosage (i.e. 20-100 mg per day) with poor oral bioavailability. Additionally, the said drug also suffers from extensive first-pass metabolism, low absorption and very low drug concentration reach the brain, especially during oral administration [12]. Thus, herein, we intend to establish the ZRS delivery efficacy (to the brain) via NLC based nasal route, which may aid in enhancing permeation and bioavailability and thereby act as an effective approach towards systemic drug delivery.

\section{MATERIALS AND METHODS}

\section{Materials}

Ziprasidone hydrochloride was obtained as a gift sample from Jubilant Generics Ltd (Mysuru, India). While, Gelucire 43/01 and $44 / 14$, was gifted by Gattefosse (France). Further, Capmul MCM, Captex 200, Cremophor EL, Sterotex FL were also obtained as a gift sample from Abitec Group, (USA). However, Span 80, PEG 400 and Oleic acid were purchased from Merck (Mumbai, India). While, Tween 80, Tween 20 and polyvinyl alcohol (PVA) were purchased from Loba Chemie Pvt. Ltd., (Mumbai, India). 


\section{Methods}

\section{Solubility studies}

In order to handpick the best suitable excipients towards formulating desired NLC, the solubility of ZRS in various lipids was ascertained. In a typical experiment, an excess amount of selected drug was mixed with selected liquid lipids with the aid of vortex mixer. The obtained mixtures were set aside for $72 \mathrm{~h}$ and followed by centrifugation for $10 \mathrm{~min}$ at 5,000 rpm. Further, $0.5 \mathrm{ml}$ of supernatant was drawn from the obtained solutions and analyzed using electronic spectral studies at $315 \mathrm{~nm}$ (UV1800, Shimadzu, Japan). Additionally, for solid lipids, the minimum amount of molten lipids required to solubilize the desired drug was quantified via visual observation wherein the formation of a clear solution of melted lipids with ZRS was considered the endpoint [13].

\section{Compatibility studies}

Differential scanning calorimetry (DSC) thermogram was obtained with Shimadzu DSC-60 calorimeter over a temperature range of 40 to $300^{\circ} \mathrm{C}$ and at a constant heating rate of $20^{\circ} \mathrm{C} / \mathrm{min}$ [14].

\section{Pseudo ternary phase diagrams construction}

Phase diagrams which are essential in optimizing the Smix ratio were constructed using aqueous titration technique. Wherein, the selected oil phase was heated and maintained at a temperature of $75^{\circ} \mathrm{C}$, to which selected Smix was added in varying ratios $(1: 9,2: 8,3: 7,4: 6,5: 5,6: 4,7: 3$, 8:2 and 9:1) so as to form homogeneous mixtures. The mixtures so obtained were then titrated against the desired aqueous phase, and the changes observed were recorded visually [15].

\section{Effect of process/formulation variables}

\section{Effect of the liquid component}

To analyze the influence of oil phase over the desired NLC's particle size, different batches of NLC were formulated with varying oil phase concentrations $(1 \%, 2 \%$, and $3 \%)$ by keeping the Smix ratio constant at 2:1 for ZRS. Selected oil phase and Smix ratios were weighed and transferred into glass vials.

Further, the samples were mixed by using a vortex mixer and heated at a temperature of $70{ }^{\circ} \mathrm{C}$ to form a homogenous mixture [16]. Particle sizes were identified visually by using the following grade system table 1.

Table 1: Grading system for visual determination of particle size

\begin{tabular}{lll}
\hline S. No. & Appearance of dissolution medium & Particle size range \\
\hline 1 & Transparent & $10 \mathrm{~nm}-200 \mathrm{~nm}$ \\
2 & Translucent & $200 \mathrm{~nm}-300 \mathrm{~nm}$ \\
3 & Fine milky & $300 \mathrm{~nm}-\mathrm{few} \mu \mathrm{m}$ \\
4 & Presence of particles & $\mathrm{ME}^{\mathrm{a}}$ \\
$\mathrm{ME}$ : Grade A, clear microemulsion & Depends on the API \\
$\mathrm{ME}^{\mathrm{b}}:$ Grade B, microemulsion & E: Emulsion \\
\hline
\end{tabular}

\section{Effect of the solid component}

To study the effect of solid lipid concentration on the formulation of desired NLCs was done by preparing NLCs by varying solid lipid ratio to liquid lipid i.e. 1:1, 1:2, 1:3. Where briefly $500 \mathrm{mg}$ of lipid phase was added to water and agitated gently with the aid of magnetic stirrer and the obtained blends were accessed visually for clear, turbid and presence of lumps in the solution [16].

\section{Formulation of NLCs containing ZRS}

The desired NLC formulations were prepared using hot homogenization, followed by rapid ultra-sonication, wherein selected quantity of desired drugs were dissolved in molten lipids and simultaneously, aqueous phase consisting of all other components were heated separately at a constant temperature. Post drug dissolution obtained blend was transferred to aqueous medium with vigorous stirring for $5 \mathrm{~min}$ at 10,000 rpm with a hot homogenization ((Polytron ${ }^{\circledR}$ PT1600E). Further, a probe sonicator was used to sonicate the obtained emulsion and desired NLC was stored in ambercolored glass vials at a temperature of $2-10{ }^{\circ} \mathrm{C}$. Additionally, to demonstrate the response surface model, Design expert software (Version 11, Stat-Ease Inc., and Minneapolis, U. S. A.) was employed. Central composite design (CCD) is used to form a second-order quadratic equation without using a complete three-level factorial experiment. A 2 factor, 2 levels central composite design was used to design the optimized procedure to formulate the desired ZRS-NLC. Wherein Oil phase and Smix were selected as independent variables and drug loading, entrapment efficiency, and particle size were considered as dependent variables. This leads to a process of optimization with a small experimental design (13 runs) [17].

\section{Evaluation of ZRS-NLCs}

\section{Drug loading and encapsulation efficiency}

The amount of ZRS loaded in relation to the lipid phase and its encapsulation efficiency was determined by centrifuging (REMI laboratory Instrument, India) a fixed volume of desired NLC for $20 \mathrm{~min}$ at $10000 \mathrm{rpm}$. The supernatant so obtained was diluted appropriately and the amount of ZRS loaded and encapsulation efficiency was quantified using UV spectrophotometric measurements at $315 \mathrm{~nm}$ (UV1800, Shimadzu, Japan) [18], using the following equations;

$$
\begin{gathered}
\text { Drug Loading (DL) }(\%)=\left(\frac{\mathrm{Wd}-\mathrm{Ws}}{\mathrm{Wn}}\right) * 100(1) \\
\text { Entrapment efficiency (EE) }(\%)=\left(\frac{\mathrm{Wd}-\mathrm{Ws}}{\mathrm{Wd}}\right) * 100(2)
\end{gathered}
$$

Where $\mathrm{W}_{\mathrm{d}}$ is the total weight of the drug is taken, $\mathrm{W}_{\mathrm{s}}$ is the weight of the drug in the supernatant and $W_{n}$ is the total weight of obtained NLC.

\section{Particle size}

Dynamic light scattering technique was used to estimate parameters (particle size and polydispersity index) of the formulated drug-loaded NLCs by using a particle size analyzer (Model Zetasizer Nano ZS, Malvern Ltd., UK) at a scattering angle of $90^{\circ}[19]$.

\section{Surface morphology}

The surface morphology of ZRS-NLC was recorded using a (Hitachi Noran System 7 scanning electron microscope (SEM), USA) [20].

\section{In vitro drug release studies in optimized ZRS loaded NLC}

The drug release studies for ZRS-NLC were accomplished using the dialysis membrane (Hi-media, Mumbai, India). In a typical experimental set-up, NLC equivalent to $100 \mathrm{mg}$ of the selected drug was measured and placed on the dialysis membrane. The bag so formed, was placed in beaker containing a fixed quantity of simulated nasal fluid (SNF) ( $\mathrm{pH}$ 6.4) i.e. $250 \mathrm{ml}$ maintained at a temperature of $37^{\circ} \mathrm{C}$. From which, samplings were carried out at predetermined intervals, during which $2 \mathrm{ml}$ of aliquots were repeatedly withdrawn from the receptor compartment and replenished with fresh buffer. Finally, the amount of ZRS released was quantified spectrophotometrically at $315 \mathrm{~nm}$ (UV1800, Shimadzu, Japan) [21]. 


\section{Ex vivo diffusion studies of optimized ZRS-NLCs}

$E x$-vivo permeation studies for both pure drug and drug-loaded NLCs were performed by using sheep nasal mucosa. Excised sheep nasal mucosa was obtained from a local butchery house followed by washing with PBS solution and isopropyl alcohol. In order to place the samples on donor and receptor components of diffusion cell, obtained nasal mucosa was sliced into small pieces with a thickness of $0.2 \mathrm{~mm}$. The mounted samples were finally stabilized using PBS treatment for $15 \mathrm{~min}$ at a temperature of $39 \pm 0.5{ }^{\circ} \mathrm{C}$. The resulting sample was then mounted on a rim of the receptor compartment with freshly filled dissolution media and the donor compartment was placed over it. Finally, the samples were considerately drawn from the respective compartments and analyzed for the amount of ZRS permeated across the tissue using UV absorbance studies at 315 nm [22].

\section{Nasal ciliotoxicity studies}

The nasal ciliotoxicity studies were performed with freshly isolated sheep mucosa, where each tissue sample was treated with isopropyl alcohol (IPA) (positive control), optimized NLCs (the formulation understudy) and PBS (negative control). The tissue samples so processed were stored in $10 \%$ formalin solution for $2 \mathrm{~h}$, followed by $\mathrm{H}$ and $\mathrm{E}$ staining for histopathological imaging [23].

\section{In vitro haemolytic toxicity study}

In order to estimate the in vitro toxicity and in vivo membrane damage, haemolytic toxicity study has to be conducted. Wherein, blood from sheep was collected in a vial coated with Ethylene Diamine Tetra Acetic Acid (EDTA) which acts as an anti-coagulant. Further, Red blood cells were separated by centrifugation followed by filtration. The absorbances of the obtained solutions $(5-500 \mu \mathrm{g} / \mathrm{ml})$ were then determined at $315 \mathrm{~nm}$ by using UV spectrophotometer. In this study Trition X 100 is used as positive control and buffer is used as a negative control [24] and the following equation was used to calculate $\%$ of lysis for each concentration.

$$
\% \text { Haemolysis }=\frac{\text { Absorbance of Test-Absorbance at } 0 \%}{\text { Absorbance at } 100 \% \text { lysis-Absorbance at } 0 \%} * 100 \text {. }
$$

\section{In vivo bio-distribution studies}

The study was conducted after obtaining approval (No. 155/PO/Re/S/99/CPCSEA) from JSS College of Pharmacy, Mysuru institutional animal ethical committee. Rats (Wister albino) weighing 180-200g were selected for bio-distribution studies, as per the guidelines set by The National Institutes of Health, all the animals were humanely treated. In order to perform bio-distribution studies, selected rats were divided into two groups. Wherein, one group received intranasal formulations, while the other received a dose which is equivalent to $2.3 \mathrm{mg} / \mathrm{kg}$ body weight intravenously via injection through the tail vein. Nevertheless, for intranasal administration, a $50 \mu \mathrm{l}$ dose was administered by holding the rats from back in slanted position from the formulation, which is equivalent to $6 \mathrm{mg} / \mathrm{ml}[25]$.

\section{Pharmacokinetic and brain distribution study}

The plasma and brain samples required for pharmacokinetic and brain distribution studies were collected by suitably anesthetizing the animals, before scarification. The typical plasma and brain sample collections involved drawing of $2 \mathrm{ml}$ of blood at different time intervals by employing cardiac puncture technique and the obtained blood samples were stored in EDTA tubes under cold conditions. However, for plasma separation, centrifugation was done for $15 \mathrm{~min}$ at $5000 \mathrm{rpm}$ for the drawn blood samples. Further, obtained plasma was mixed with internal standard and $2 \mathrm{ml}$ of acetonitrile was added to bring about protein precipitation. Postprecipitation, the plasma samples were further thoroughly mixed and centrifuged for a short period of time ( $5 \mathrm{~min}$ at $4000 \mathrm{rpm}$ ). To finish, supernatant obtained from the centrifuged solutions was collected and prior to analysis the obtained samples were dried at ambient temperatures and reconstituted with the selected mobile phase and filtered through a membrane filter.

Post blood collection, animals were decapitated immediately and the skull was slit to excise the brain carefully. In order to get free from attached blood vessels, the obtained brain tissue was quickly washed with saline water and marked with the help of a filter paper and stored in PBS. The stored tissues were then homogenized for 1 min at $10000 \mathrm{rpm}$ followed by centrifugation and the obtained samples were analyzed by using HPLC. Post analysis of various pharmacokinetic parameters was calculated by using formulas that were cited elsewhere [26].

\section{RESULTS AND DISCUSSION}

\section{Solubility studies}

The selection of right excipient towards the effective formulation of NLC is mostly dependent on the solubility of the desired drug (in actual excipients). Accordingly, data obtained from solubility studies of ZRS in various components were depicted in fig. 1-2. All the excipients chosen had shown a better solubility for ZRS. While, the initial selection of lipids few liquid lipids were obtained and solubility of ZRS was ascertained in the same (fig. 1), from the results obtained it was evident that Capmul MCM had shown maximum solubility of ZRS and was selected as a liquid lipid to formulate the desired NLCs.

Quantity of the obtained solid lipids required to solubilize $20 \mathrm{mg}$ of ZRS was calculated and depicted in fig. 2 . From the results, it was evident that Gelucire 43/01 had shown a better solubility when compared with other lipids. Further, results also suggested that ZRS had shown more solubility in liquid lipids when compared with solid lipids. Additionally, it was found that Gelucire 43/01 had formed a homogeneous mixture when combined with Capmul MCM without any phase separation for $24 \mathrm{~h}$, suggesting that the selected liquid lipid has completely entrapped in solid lipid which is the initial step in formulating the desired NLCs. On basing these solubility studies Capmul MCM and Gelucire 43/01 were selected as the lipid phase for further studies [13].

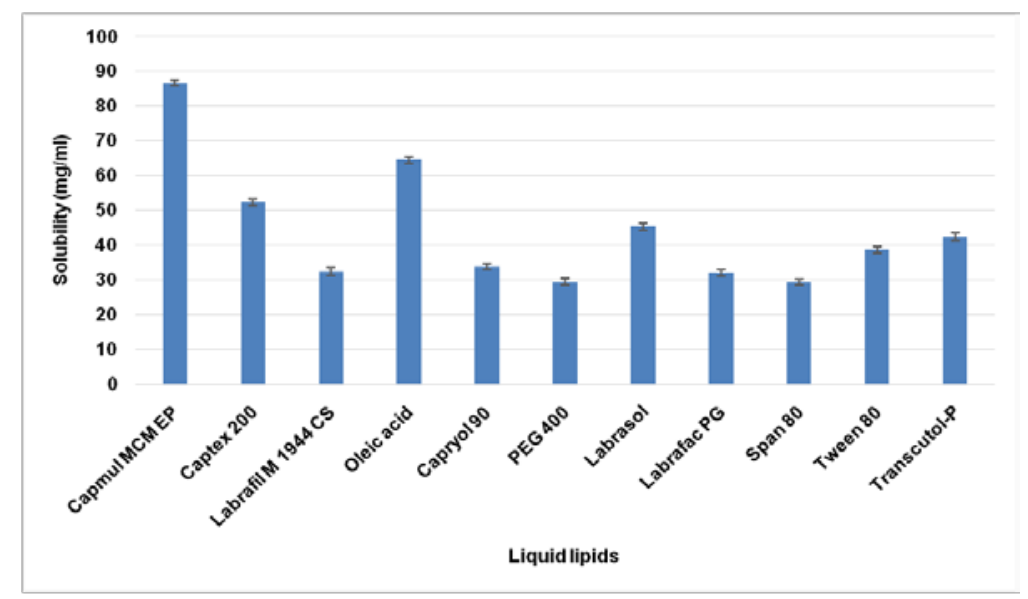

Fig. 1: The solubility of ZRS in liquid lipids; mean $\pm S D, n=3$ 


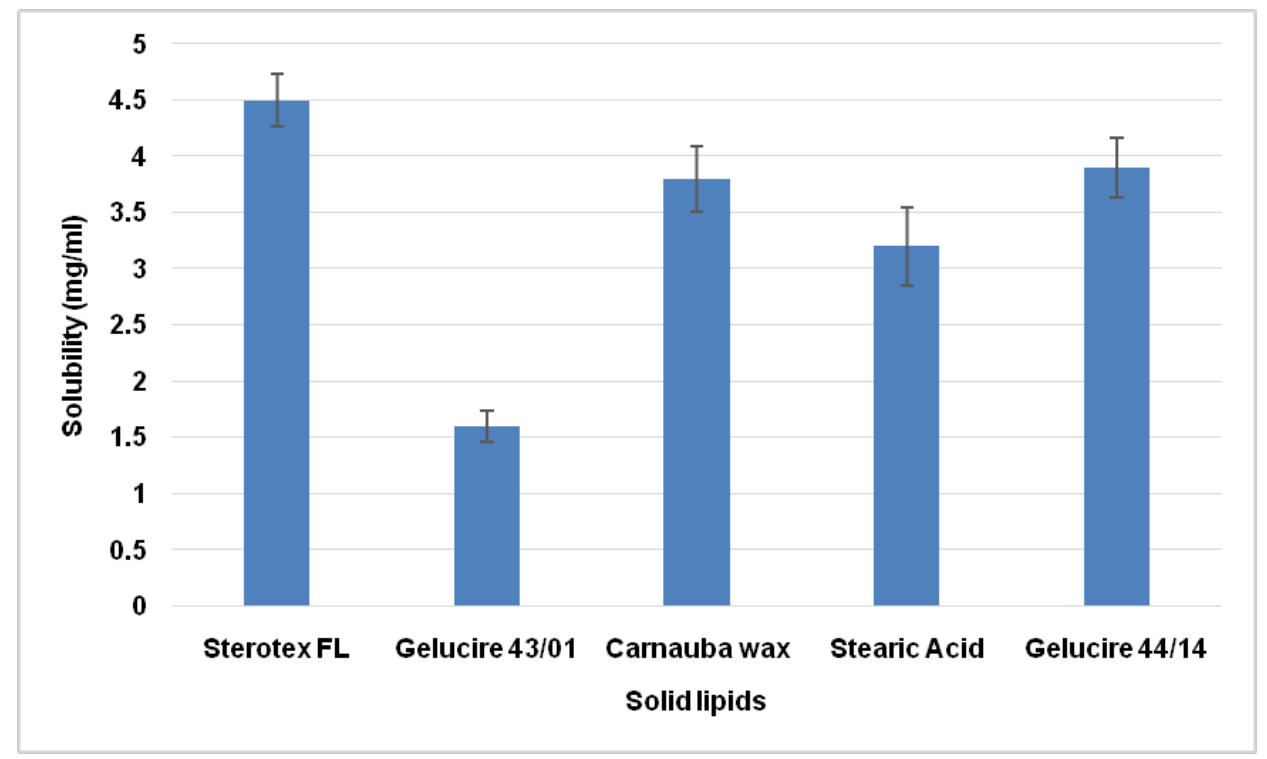

Fig. 2: The solubility of ZRS in solid lipids; mean $\pm S D, n=3$

\section{Compatibility study}

\section{DSC studies}

Thermograms obtained from DSC analysis for both pure ZRS and ZRS physical mixture were depicted in fig. 3 respectively. Wherein, pure ZRS thermogram had shown a sharp endotherm peak at $236.68{ }^{\circ} \mathrm{C}$, which is in agreement with ZRS's melting range. While, the physical mixture displayed two peaks, first at $39.28^{\circ} \mathrm{C}$ (corresponding to the oil phase), where the drug may be molecularly dispersed in corresponding lipids. While, the second peak at $237.48{ }^{\circ} \mathrm{C}$ may be attributed to pure ZRS, indicating the absence of undesirable druglipid interactions [27].

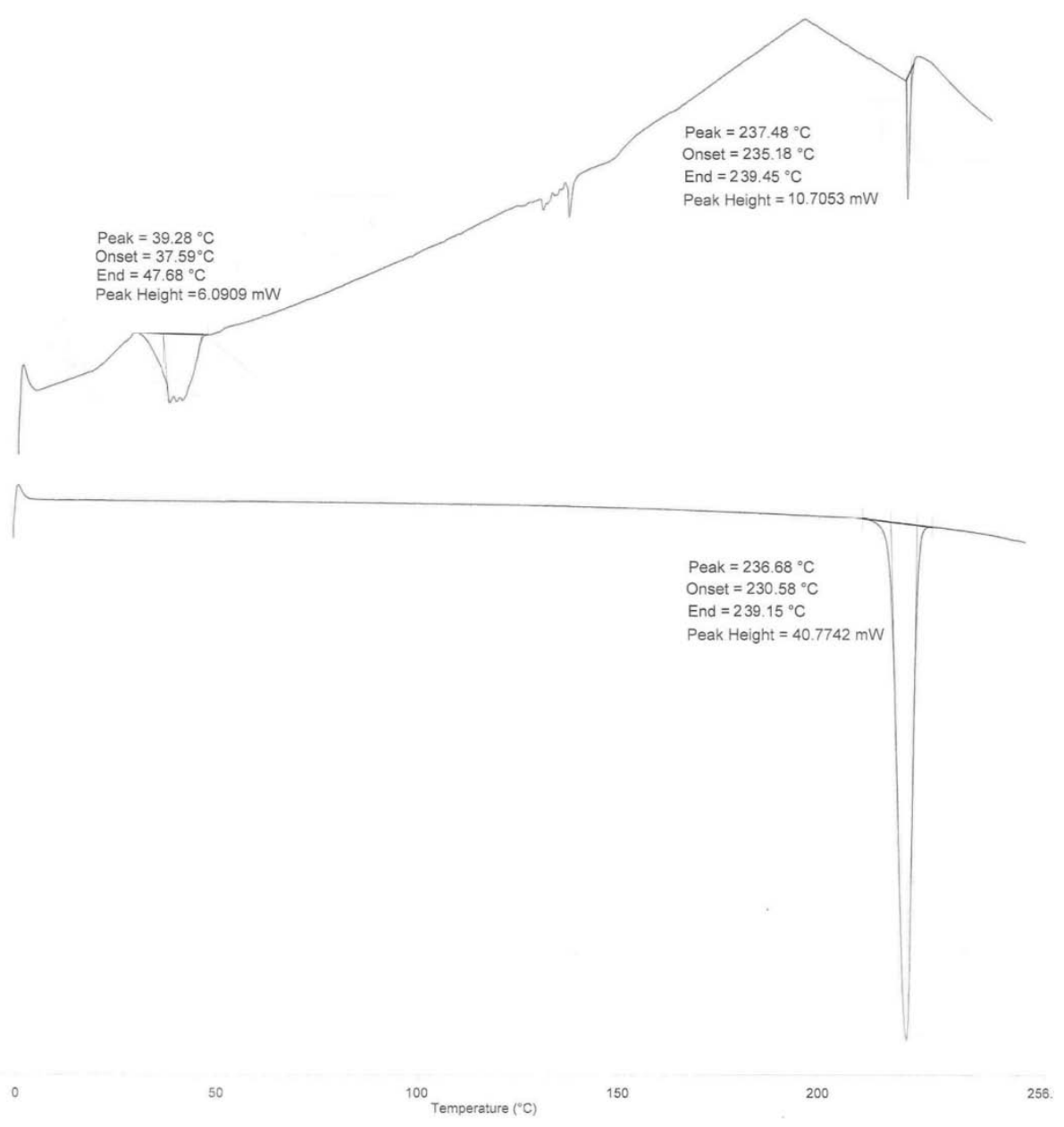

Fig. 3: DSC thermogram of ZRS and physical mixture 


\section{Construction of pseudo-ternary phase diagrams}

The (drug excipient) formulations, which remained as nanoemulsions (when dispersed) were subjected to further studies. Nanoemulsions composed of oil phase (Capmul MCM and Gelucire 43/01), Labrasol as surfactant and Transcutol-p as co-surfactant, which were selected for further optimization. As can be seen from fig. 4(A-D)-5(A-D), the nanoemulsion formation area increases with an increase in the Smix ratio and found to be highest at 2:1. Consequently, 2:1 Smix ratio was maintained for further optimizations [28].
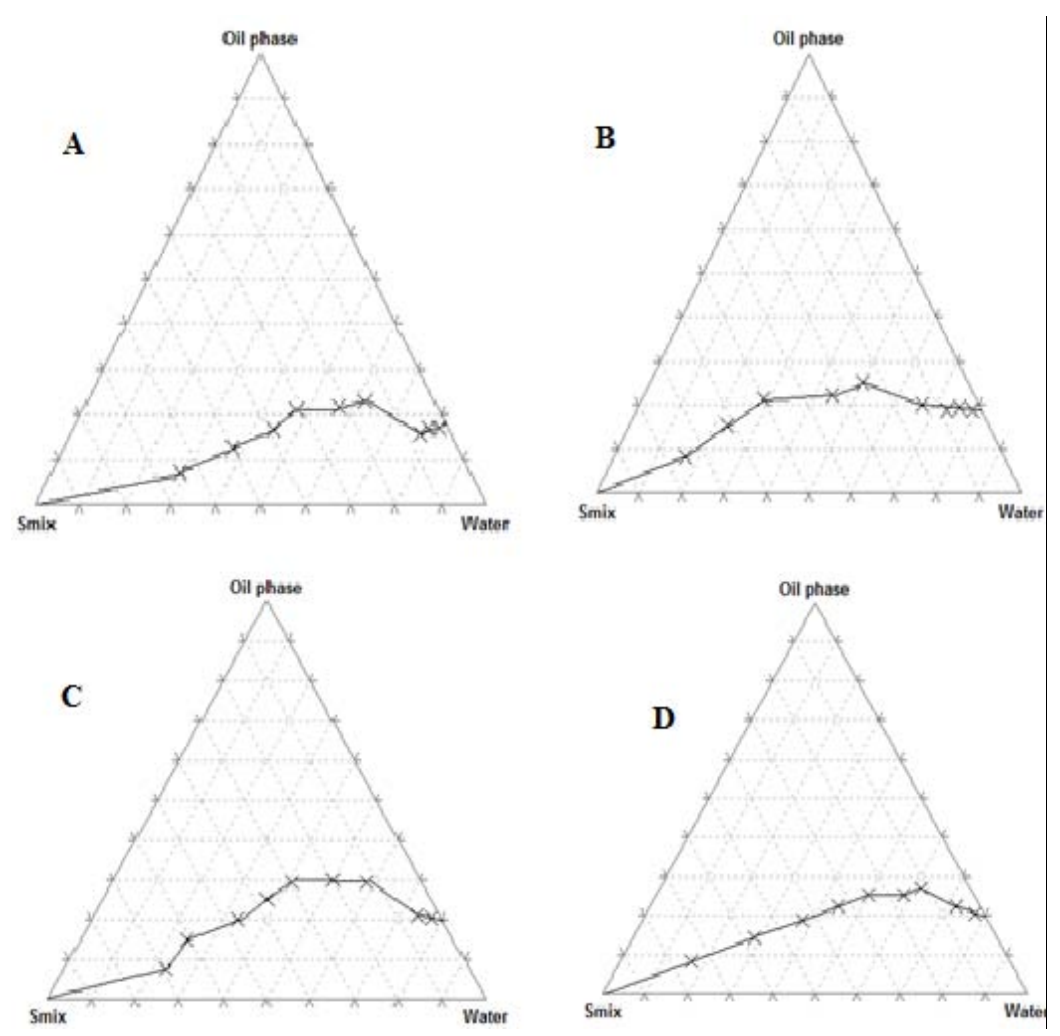

Fig. 4 (A-D): Pseudo-ternary phase diagram for a group I
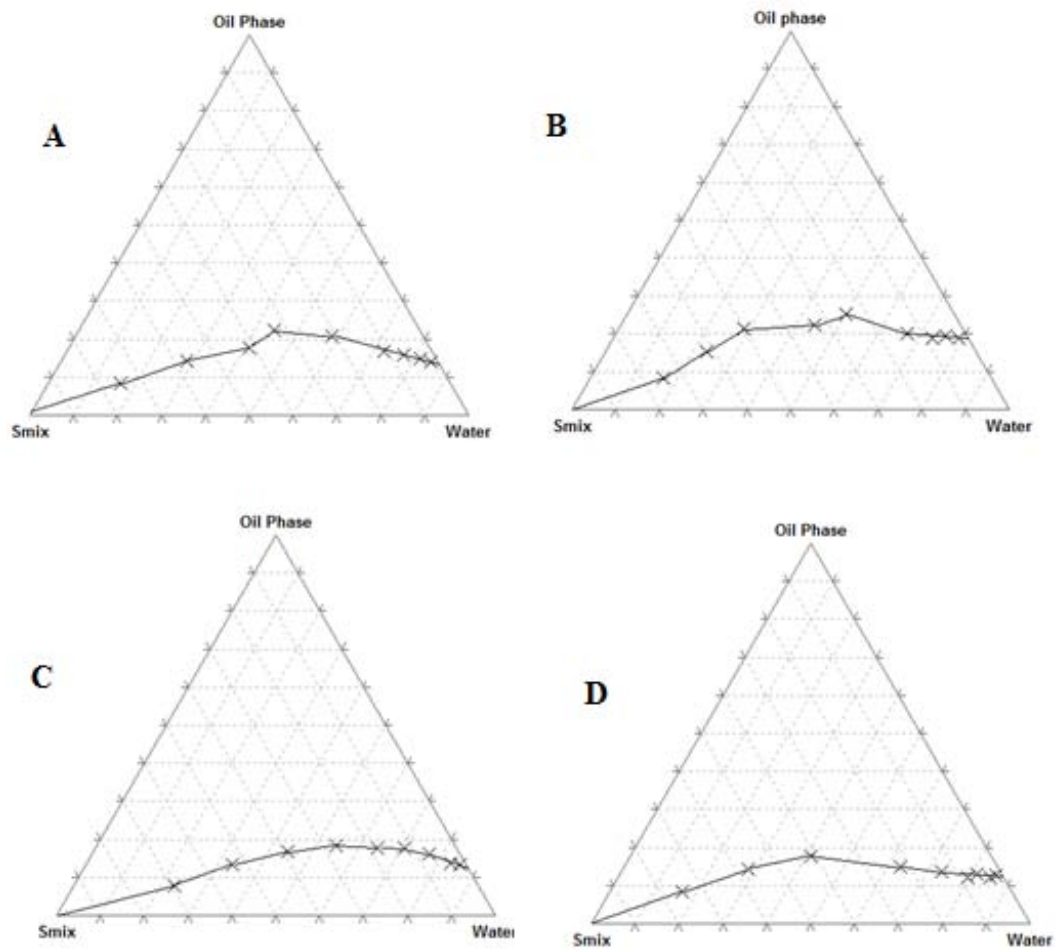

Fig. 5 (A-D): Pseudo-ternary phase diagram for group II 


\section{Effect of process/formulation variables}

\section{Effect of liquid lipid concentration}

When the concentration of the oil phase is increased above $1 \%$ there was a substantial enhancement in particle size. Obtained results suggested that an increase in the ratio of oil phase to Smix has increased particle size. Therefore, $1 \%$ of lipid concentration was considered as optimum concentration for further batches (table 2).

\section{Effect of the amount of solid lipid on ease of emulsification}

Obtained results revealed that formulation consisting of 1:1 ratio of lipid phase has produced translucent solutions, while $1: 2$ and 1:3 formulations had shown milky and turbid appearance as depicted in table 3. An increase in the solid lipid content has increased emulsification time, which is due to the melting point of Gelucire $43 / 01$. An increase in the weight ratio of solid lipids led to globules with increased size. Therefore, to achieve rapid dissolution rates, 1:1 lipid ratio was selected for further development of NLC.

Table 2: Effect of liquid lipid concentration

\begin{tabular}{lll}
\hline S. No. & Concentration $(\mu \mathbf{g} / \mathbf{m l})$ & Visual reading \\
\hline 1 & $1 \%$ & Translucent \\
3 & $2 \%$ & Fine milky \\
3 & $3 \%$ & Fine milky \\
\hline
\end{tabular}

Table 3: Effect of solid lipid on ease of emulsification

\begin{tabular}{llll}
\hline S. No. & Oil: solid lipid & Visual reading & Result \\
\hline 1 & $1: 1$ & Translucent & ME $^{\mathrm{a}}$ \\
2 & $1: 2$ & Fine milky & E \\
3 & $1: 3$ & Turbid & $\mathrm{E}$ \\
\hline
\end{tabular}

Table 4: Variables in the central composite design of ZRS-NLC

\begin{tabular}{llll}
\hline Independent factors & & Design level & \\
\hline Uncoded & Coded & Uncoded & Coded \\
\hline Oil Phase (\%) & $\mathrm{X}_{1}$ & 5 & -1 \\
& & 7.5 & 0 (Center point) \\
& & 10 & -1 \\
$\mathrm{~S}_{\text {mix }}(\%)$ & $\mathrm{X}_{2}$ & 20 & 0 (Center point) \\
& & 35 & 50 \\
\hline
\end{tabular}

Table 5: Observed responses in the central composite design of ZRS-NLC

\begin{tabular}{|c|c|c|c|c|c|}
\hline Formulation code & Oil phase & $S_{\text {mix }}$ & Drug loading (\%) & EE (\%) & Particle size (nm) \\
\hline ZRS1 & 7.5 & 56.2132 & 18.32 & 70.83 & 119.62 \\
\hline ZRS2 & 7.5 & 35 & 15.62 & 60.85 & 140.25 \\
\hline ZRS3 & 7.5 & 13.7868 & 8.92 & 41.2 & 165.56 \\
\hline ZRS4 & 10 & 50 & 21.85 & 81.34 & 145.24 \\
\hline ZRS 5 & 3.96447 & 35 & 22.85 & 80.52 & 110.34 \\
\hline ZRS 6 & 7.5 & 35 & 15.62 & 60.85 & 140.25 \\
\hline ZRS 7 & 7.5 & 35 & 15.62 & 60.85 & 140.25 \\
\hline ZRS8 & 11.0355 & 35 & 19.86 & 79.25 & 165.75 \\
\hline ZRS 9 & 10 & 20 & 12.95 & 62.25 & 167 \\
\hline ZRS 10 & 7.5 & 35 & 15.62 & 60.85 & 140.25 \\
\hline ZRS 11 & 5 & 50 & 25.52 & 83.62 & 98.64 \\
\hline ZRS12 & 5 & 20 & 5.65 & 36.92 & 90.28 \\
\hline ZRS 13 & 7.5 & 35 & 15.62 & 60.85 & 140.25 \\
\hline
\end{tabular}

\section{Formulation of ZRS loaded NLC}

The ZRS-NLC consisting of Gelucire 43/01+Capmul MCM (1:1 ratio), Labrasol and Transcutol-p were used as surfactants and cosurfactants in 2:1 ratio was formulated via homogenization (under hot) followed by rapid-ultra sonication. The as-prepared, ZRS-NLC formulation existed as pale pink colour dispersions.

Central composite design (table 4-5) was employed towards formulating NLCs at 2 factors and 2 levels, with $\mathrm{X}_{1}$ (Oil Phase) and $\mathrm{X}_{2}$ $\left(S_{\text {mix }}\right)$ as independent variables, while levels- 1 and +1 were chosen as low and high, whereas 0 was chosen as the centre point [29].

\section{Effect of dependent variables on responses}

\section{Drug loading and entrapment efficiency}

Effect of Independent variables (under investigation) over drug loading and entrapment efficiency (as established by central composite design) was presented in table 5 . As can be seen, both Oil phase and $S_{\text {mix }}$ displayed a monotonic relationship on entrapment efficiency and drug loading (fig. 6). Accordingly, with an increase in the concentration of the oil phase, there was a significant increment in both responses, thereby reducing the escaping nature of the drug into the external phase. Moreover, for optimized formulation (ZRS14), entrapment efficiency was found to be $83.53 \%$ with a drug loading of $25.19 \%$, while the rest of the drug remained free in the dispersion media (16.47\% of the loaded $25.19 \%$ ). Thus, $16.47 \%$ of loaded $25.19 \%$ drug (present in dispersion media) was considered as the loading dose. While $83.53 \%$ of loaded $25.19 \%$ (amount of drug entrapped in NLC) was considered to be the maintenance dose (towards sustained delivery). Further, the desired polynomial equations, showed a good fit of responses at different concentrations (table 6), thereby supporting the significance of the said model [29].

Drug loading (R1) $=-104.73066+21.79728$ Oil phase +5.31367 Smix 0.936676 Oil phase $*$ Smix-1.02546 Oil phase ${ }^{2}-0.031745$ Smix $^{2}+$ 0.041270 Oil phase ${ }^{2 *}$ Smix +0.003493 Oil phase $*$ Smix $^{2}$

Entrapment efficiency (R2) $=-171.85340+31.54012$ Oil phase+ 12.36058 Smix-1.91253 Oil phase* Smix-0.783340 Oil phase ${ }^{2}-0.095695$ Smix $^{2}+0.063698$ Oil phase ${ }^{2 *}$ Smix +0.011043 Oil phase* Smix $^{2}$

\section{Particle size}

Effect of dependent variables over particle size as depicted in table 5. Results suggested that both variables have a progressive impact over particle size, while the effect is negative, with Smix as shown in (fig. 6). 
Further, an increase in concentrations of oil phase leads to the formation of aggregates, which in turn increases the particle size from 98.7 to 220.6 (as shown in table 5). With an increase in the concentration of Smix, there is a rapid decrement in particle size. Further, the desired polynomial equations, showed a good fit of responses at different concentrations (table 6), thereby supporting the significance of the said model. Further, for ZRS-14, average particle size was found to be around
$106.4 \mathrm{~nm}$ and particle size distribution of optimized formulation was depicted in fig 7. The results obtained infers that the selected excipients had led to the effective development of desired NLC [29].

Particle Size (R3) $=+140.25+19.590$ il phase-16.24 Smix-7.53 Oil phase * Smix-4.86 Oil phase ${ }^{2}-2.59$ Smix $^{2}+12.89$ Oil phase ${ }^{2} *$ Smix+11.240il phase $*$ Smix $^{2}$

Table 6: Summary of ANOVA for responses of ZRS-NLC

\begin{tabular}{|c|c|c|c|c|c|c|}
\hline \multicolumn{7}{|c|}{ Drug loading (\%) } \\
\hline Source & Sum of squares & df & Mean square & F value & P value & Remarks \\
\hline Model & 354.56 & 7 & 50.65 & 127.91 & $<0.0001$ & Significant \\
\hline A-Oil Phase & 4.47 & 1 & 4.47 & 11.29 & 0.0201 & \\
\hline $\mathrm{B}-\mathrm{S}_{\operatorname{mix}}$ & 44.18 & 1 & 44.18 & 111.56 & 0.0001 & \\
\hline $\mathrm{AB}$ & 30.09 & 1 & 30.09 & 75.97 & 0.0003 & \\
\hline$A^{2}$ & 47.71 & 1 & 47.71 & 120.47 & 0.0001 & \\
\hline $\mathrm{B}^{2}$ & 10.85 & 1 & 10.85 & 27.39 & 0.0034 & \\
\hline$A^{2} B$ & 29.94 & 1 & 29.94 & 75.60 & 0.0003 & \\
\hline $\mathrm{AB}^{2}$ & 7.72 & 1 & 7.72 & 19.49 & 0.0069 & \\
\hline Residual & 1.98 & 5 & 0.3960 & & & \\
\hline Lack of fit & 1.98 & 1 & 1.98 & & & \\
\hline Pure error & 0.0000 & 4 & 0.0000 & & & \\
\hline Cor Total & 356.54 & 12 & & & & \\
\hline \multicolumn{7}{|c|}{ Entrapment efficiency (\%) } \\
\hline Model & 2531.08 & 7 & 361.58 & 245.85 & $<0.0001$ & Significant \\
\hline A-Oil Phase & 0.8064 & 1 & 0.8064 & 0.5483 & 0.4923 & \\
\hline $\mathrm{B}-\mathrm{S}_{\operatorname{mix}}$ & 438.97 & 1 & 438.97 & 298.47 & $<0.0001$ & \\
\hline $\mathrm{AB}$ & 190.58 & 1 & 190.58 & 129.58 & $<0.0001$ & \\
\hline$A^{2}$ & 568.26 & 1 & 568.26 & 386.38 & $<0.0001$ & \\
\hline $\mathrm{B}^{2}$ & 58.38 & 1 & 58.38 & 39.69 & 0.0015 & \\
\hline$A^{2} B$ & 71.32 & 1 & 71.32 & 48.50 & 0.0009 & \\
\hline $\mathrm{AB}^{2}$ & 77.17 & 1 & 77.17 & 52.47 & 0.0008 & \\
\hline Residual & 7.35 & 5 & 1.47 & & & \\
\hline Lack of fit & 7.35 & 1 & 7.35 & & & \\
\hline Pure error & 0.0000 & 4 & 0.0000 & & & \\
\hline Cor Total & 2538.44 & 12 & & & & \\
\hline \multicolumn{7}{|c|}{ Particle size (nm) } \\
\hline Model & 6855.29 & 7 & 979.33 & 10.84 & 0.0092 & Significant \\
\hline A-Oil Phase & 1535.13 & 1 & 1535.13 & 16.99 & 0.0092 & \\
\hline $\mathrm{B}-\mathrm{S}_{\operatorname{mix}}$ & 1055.24 & 1 & 1055.24 & 11.68 & 0.0189 & \\
\hline $\mathrm{AB}$ & 226.80 & 1 & 226.80 & 2.51 & 0.1739 & \\
\hline$A^{2}$ & 164.27 & 1 & 164.27 & 1.82 & 0.2353 & \\
\hline $\mathrm{B}^{2}$ & 46.55 & 1 & 46.55 & 0.5154 & 0.5050 & \\
\hline$A^{2} B$ & 332.42 & 1 & 332.42 & 3.68 & 0.1132 & \\
\hline $\mathrm{AB}^{2}$ & 252.66 & 1 & 252.66 & 2.80 & 0.1553 & \\
\hline Residual & 451.65 & 5 & 90.33 & & & \\
\hline Lack of fit & 451.65 & 1 & 451.65 & & & \\
\hline Pure error & 0.0000 & 4 & 0.0000 & & & \\
\hline Cor Total & 7306.94 & 12 & & & & \\
\hline
\end{tabular}

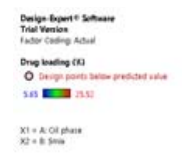

A

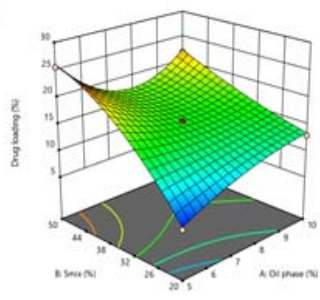

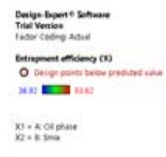

B

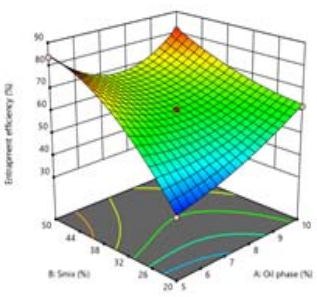

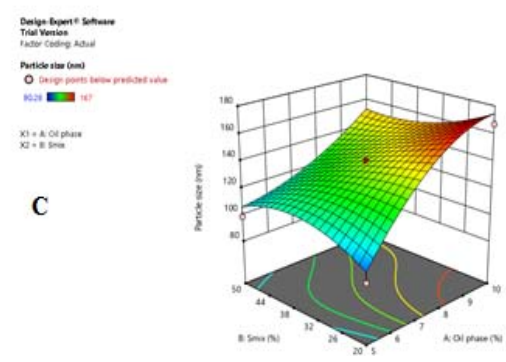

Fig. 6: Three-dimensional response surface plot depicting the impact of the oil phase and $S_{\text {mix }}$ on (A) drug loading (B) entrapment efficiency and (C) particle size of ZRS-NLCs 


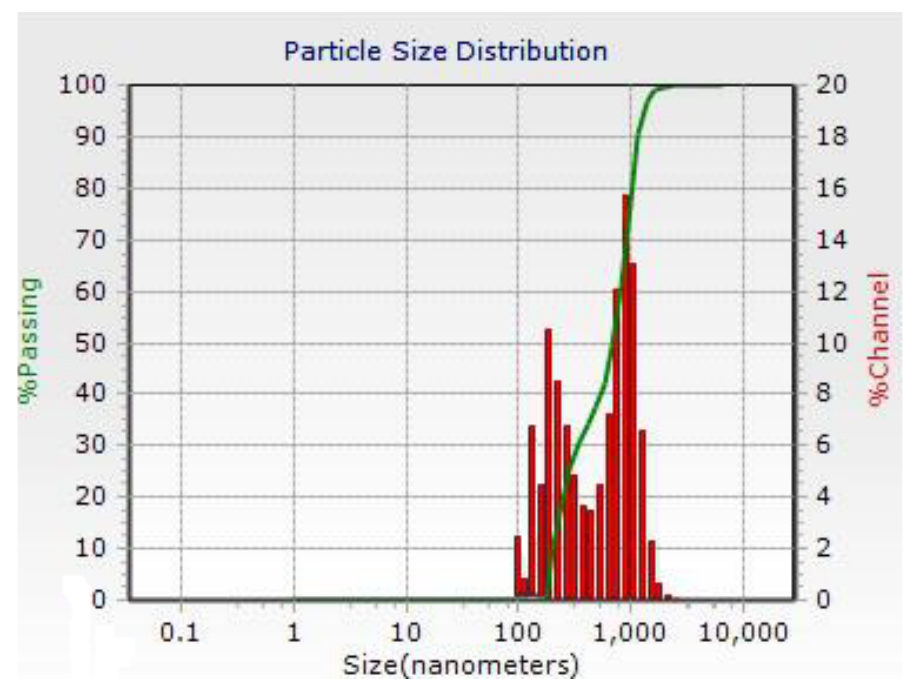

Fig. 7: Particle size distribution of optimized ZRS-NLC

\section{Optimized formulation}

Optimized formulation (ZRS-14) was obtained by outlining responses (required limits) as depicted in table 7. Design expert software was used to calculate the required specifications in order to formulate desired NLC with combinations of variables. Further, to predict the practicability and validity of the obtained model obtained, results were overlapped with predicted values [29].

Table 7: Central composite design enabled ZRS-NLC (optimized formulation) obtained via independent variables and their effects on dependent responses

\begin{tabular}{lllllll}
\hline Value & Oil phase (\%) & S $_{\text {mix }}(\%)$ & Entrapment efficiency (\%) & Drug loading (\%) & Particle size (nm) & Desirability \\
\hline Predicted & 5.08 & 50 & 83.53 & 25.51 & 107 \\
Actual & 5.1 & 50 & 83.49 & 25.14 & 106 \\
\% Error & 0.02 & 0 & 0.04 & 0.37 & 0.991 \\
\hline
\end{tabular}

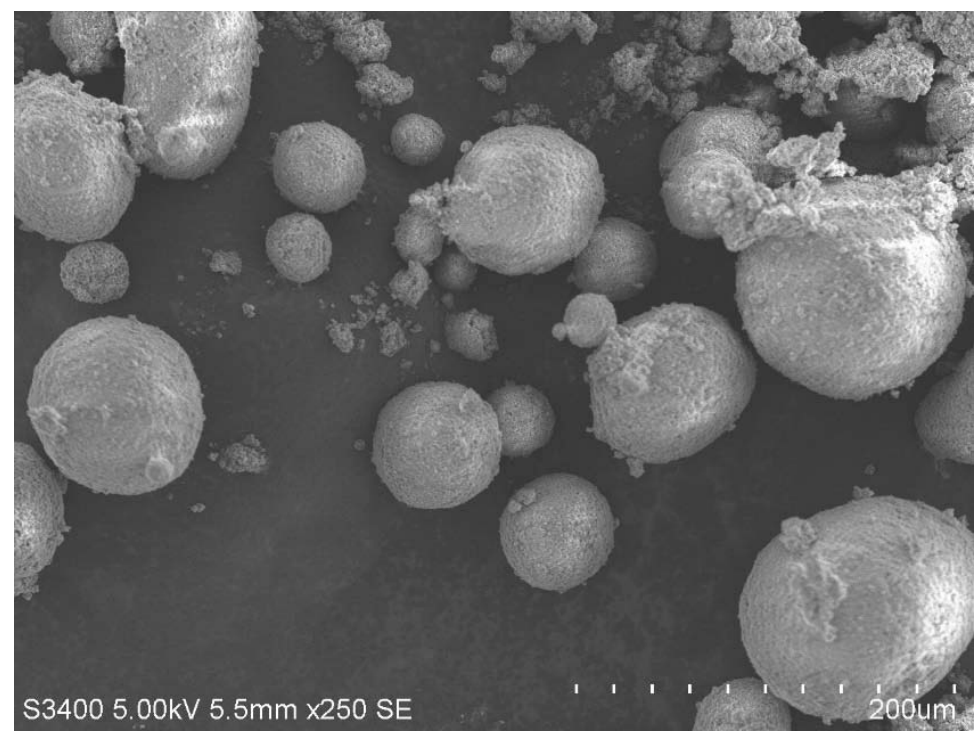

Fig. 8: Surface morphology of optimized ZRS-NLC

\section{Surface morphological behaviours of ZRS-NLC}

The SEM photomicrograph of ZRS-NLC (fig. 8) supported that particles were spherical in shape and near homogeneous with relatively smoother surfaces [20].

\section{A comparative account of drug release via in vitro studies}

ZRS-NLC drug release profile was compared with pure ZRS (fig. 9) supported that, ZRS-NLC displayed sustained drug release.
Accordingly, the drug release from pure ZRS was rapid (98.45 over $8 \mathrm{~h}$ ) due to initial rapid ZRS release from the NLC surface, in contrast to prolonged-release from ZRS-NLC $(26.72-80.34 \%$ over $24 \mathrm{~h}$ ), thereby supporting the sustained delivery of the desired drug and hence, the effectiveness of the developed formulation. Additionally, the ZRS release pattern from the optimized formulation was bi-phasic, associated with an initial short-timed $(2 \mathrm{~h})$ burst release, followed by a long term $(24 \mathrm{~h})$ sustainedrelease [21]. 


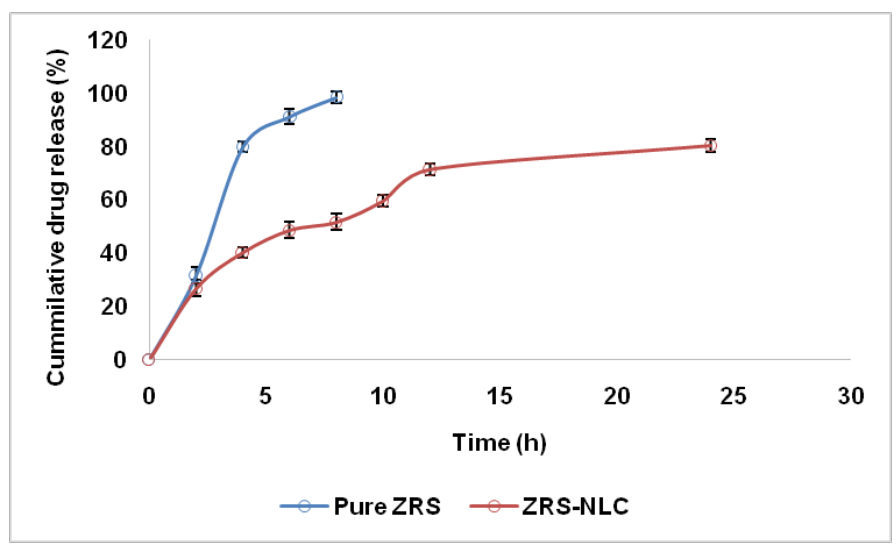

Fig. 9: A comparative in vitro drug release profile of pure ZRS and ZRS-NLC; mean $\pm S D, n=3$

\section{Cumulative drug permeation measurements via ex-vivo diffusion studies}

The data obtained from the permeation studies of pure ZRS and optimized ZRS-NLC with sheep nasal mucosa revealed that ZRS-NLC had shown significant enhancement in permeation (in contrast to pure ZRS). Accordingly, about $77.25 \pm 0.19 \%$ of ZRS was permeated from the optimized NLC formulation, while the same was around $32.52 \pm 0.19 \%$ for the pure ZRS (at the end of the same time interval $(6 \mathrm{~h})$ ). Consequently, a steady-state flux of $1.26 \mu \mathrm{g} / \mathrm{cm}^{2} / \mathrm{h}$ was observed in pure ZRS, whereas, for ZRS-NLC, it was recorded as 5.04 $\mu \mathrm{g} / \mathrm{cm}^{2} / \mathrm{h}$. The observed higher flux may be attributed to the presence of permeation enhancing surfactants in ZRS-NLC Moreover, the relatively higher lipophilicity of ZRS allows significantly better permeation through the nasal mucosa [30].

\section{Nasal ciliotoxicity studies}

In order to assess the toxicity in the selected excipients employed towards the development of ZRS-NLCs ciliotoxicity studies were performed. Further, the obtained pictograms had revealed that tissue samples treated with the positive control (IPA) had shown a severe impact on the sample leading to nasal mucosa damage, mucosal shrinkage, and loss of both cilia and epithelial cells. However, samples treated with negative control (PBS) had shown intact epithelial layers with zero damage to nasal cilia cells. However, the nasal mucosa samples treated with optimized ZRS-NLC showed no adverse effects (fig. 10). Accordingly, the nasal ciliotoxicity studies established that all the excipients involved in the formulation of ZRS-NLC were safe enough for nasal administration [21].

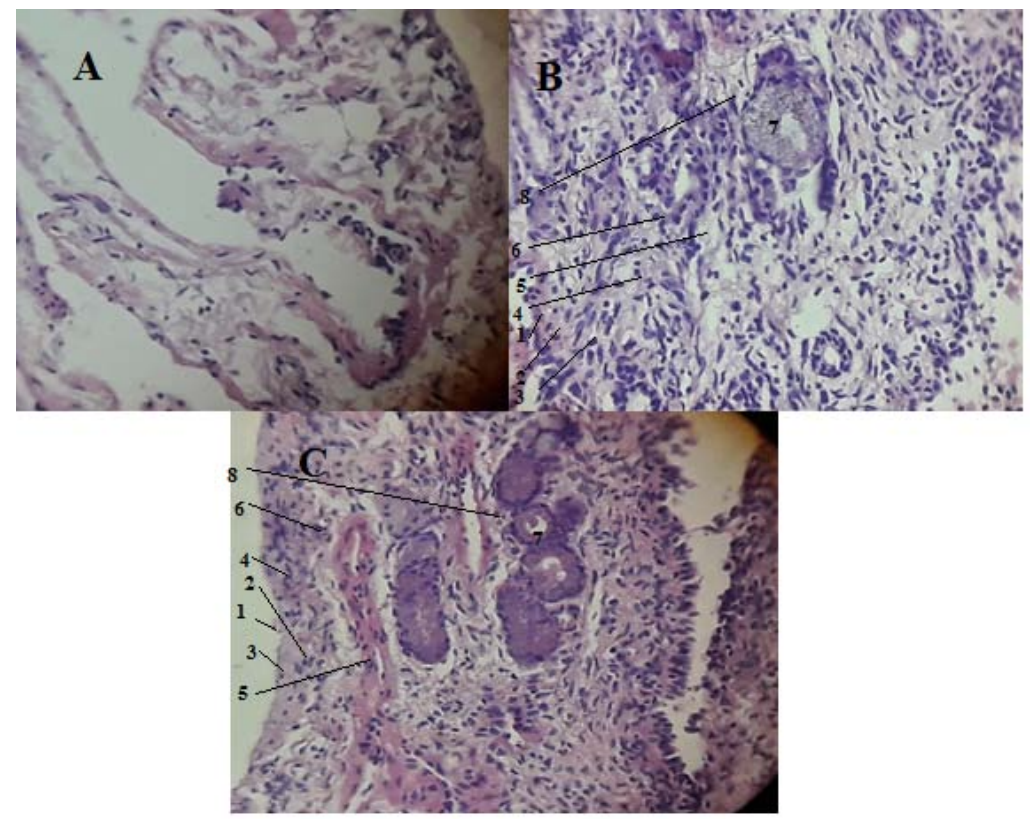

1. Respiratory epithelium 2. Nuclei of Supportive cell 3. Goblet cells 4 . Ducts of olfactory 5 . Nuclei of Basal Cells 6 . Nuclei of olfactory cells 7 . Olfactory glands 8. Lamina Propria

Fig. 10: Histopathological sections of sheep nasal mucosa treated with (a) IPA (b) ZRS-NLC and (c) PBS for nasal ciliotoxicity studies

\section{In vitro haemolytic toxicity study}

The formulated ZRS-NLCs upon intranasal administration will enter into the systemic circulation where there is a chance of damaging the red blood cells. To ascertain the safety of the formulated NLC In vitro haemolytic toxicity studies were carried out by formulating a blank and drug-loaded NLC, which were incubated by isolated RBCs. Results (table 8) suggested that both formulated NLCs were evaluated at a concentration range of $0-500 \mu \mathrm{g} / \mathrm{ml}$, which showed a negligible haemolytic effect (0.196-2.857\%), which were within the prescribed limit $(<5 \%)$. In order to validate the method Triton X 100, a wellknown haemolytic agent was taken as a positive control [31]. 
Table 8: In vitro hemolytic study of ZRS-NLC

\begin{tabular}{lll}
\hline Concentration $(\boldsymbol{\mu g} / \mathbf{m l})$ & \multicolumn{2}{l}{ Percent haemolysis (\%)* } \\
\cline { 2 - 3 } & Blank NLC & $0.375 \pm 0.01$ \\
\hline 5 & $0.196 \pm 0.02$ & $0.635 \pm 0.05$ \\
10 & $0.395 \pm 0.01$ & $0.952 \pm 0.02$ \\
25 & $0.824 \pm 0.02$ & $1.452 \pm 0.02$ \\
50 & $1.356 \pm 0.03$ & $1.785 \pm 0.03$ \\
100 & $1.789 \pm 0.05$ & $1.898 \pm 0.01$ \\
500 & $1.852 \pm 0.02$ & $2.265 \pm 0.05$ \\
Absorbance at 100\% lysis & $2.857 \pm 0.05$ & \\
Absorbance at $0 \%$ lysis & $0.848 \pm 0.01$ & $0.297 \pm 0.05$ \\
$100 \%-0 \%$ & $0.551 \pm 0.02$ & \\
\hline
\end{tabular}

*mean \pm SD, $\mathrm{n}=3$

Quantification of drug concentration via in vivo bio-distribution studies

Pharmacokinetic profiles of both plasma and brain (concentration against time) for ZRS-NLC delivered through various routes are summarized, and representative chromatogram of animal study sample at $360^{\text {th }}$ min was depicted in (fig. 11-12). The distribution studies revealed that the concentration of ZRS in the brain following IN administration was significantly higher at all-time points. Nevertheless, when IV route was followed, the concentration of ZRS was highest in blood plasma (at all-time points) and lowest in the brain. The obtained results supported that when the formulation was administered through nasal route, amount of drug distributed through systemic circulation was lower when compared with the intravenous route, thereby indicating a potential way to deliver the desired drug to the brain via nasal route. Further, the obtained blood to brain ratio for ZRS administered intranasally was significantly higher $(1.63 \pm 0.31)$, which supports increased and sustained retaining of ZRS at the brain $(\sim 10$ times higher, than IV administration). Moreover, lower $\mathrm{T}_{\max }$ (time taken to attain maximum concentration) value of $10 \mathrm{~min}$ in the brain, compared 25 min in plasma suggested that following nasal administration there is direct transport from nose to brain. Additionally, the Dug targeting efficiency (DTE) and direct transport percentage (DTP) value for ZRS-NLC via nasal administration was found to be $476.76 \pm 9.53$ and $89.85 \pm 7.85$, supporting higher targeting efficiency. The lesser nasal bioavailability of ZRS in plasma may be attributed to the detail that, whenever a drug is administered through nasal route it uses both olfactory and trigeminal nerves for its transport, thereby successfully bypassing the BBB [32].

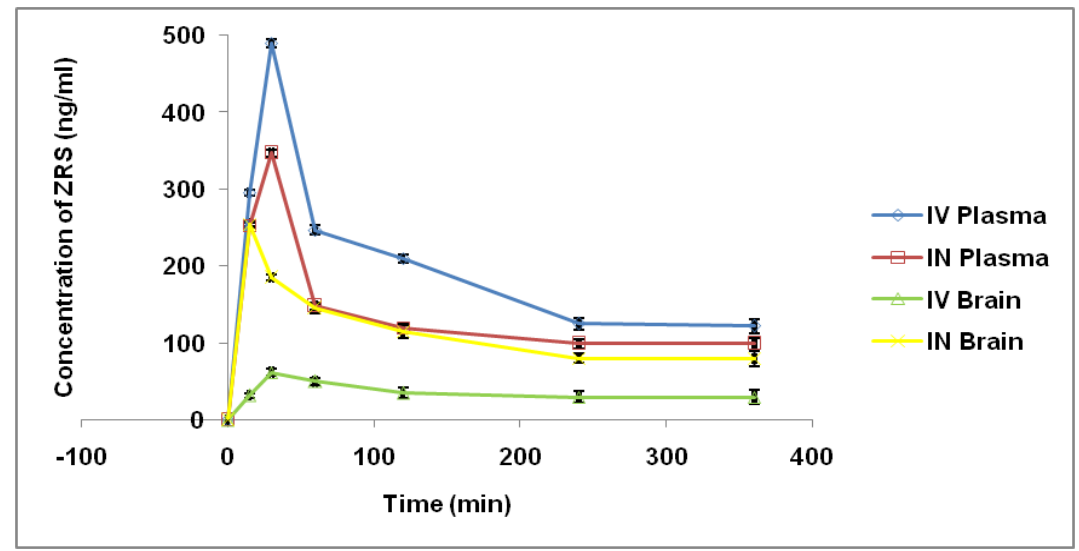

Fig. 11: Mean concentration-time profile after IN and IV administration in both plasma and brain; $m e a n \pm S D, n=3$

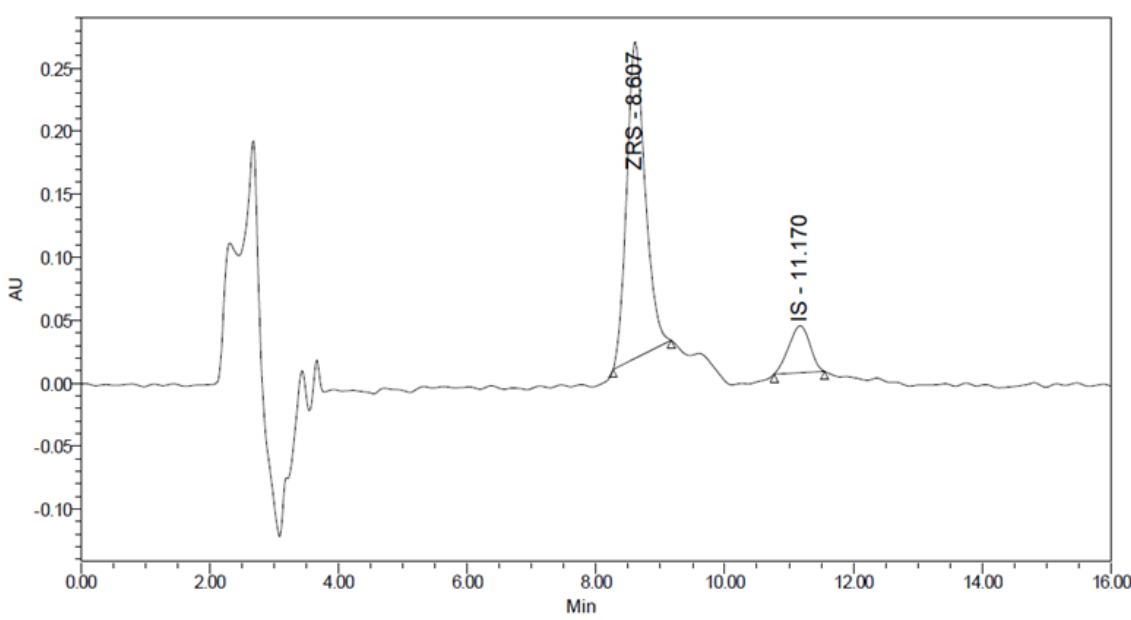

Fig. 12: Representative chromatogram of the animal study sample of $\mathrm{ZRS}$ at $360^{\text {th }} \mathrm{min}$ 


\section{CONCLUSION}

The central composite design was used to formulate desired ZRSNLCs, furnishing with oil phase and Smix to obtain NLC with minimum particle size and maximum drug loading and entrapment efficiency. The present research suggested an enhanced brain targeting of ZRS by nasal route. Moreover, the observed high DTE and DTP values for ZRSNLC suggested that formulated ZRS-NLC had by-passed BBB. The research also supports that optimized ZRS-NLC can permeate through nasal mucosa without any structural damages. To sum up, the present findings support that ZRS-NLC could be a promising alternative approach for brain targeting via nasal route.

\section{ACKNOWLEDGMENT}

The authors express their gratitude to the JSS Academy of Higher Education and Research and JSS College of Pharmacy, Mysuru, for providing necessary support in due course of the work.

\section{AUTHORS CONTRIBUTIONS}

Praveen Sivadasu was responsible for the study concept, design, and acquisition of data; Gowda D. V has monitored the entire study and helped in drafting the manuscript; Siddaramaiah and Hemalatha S had helped in drafting and revised the manuscript.

\section{CONFLICT OF INTERESTS}

The author confirms that this article content has no conflict of interest.

\section{REFERENCES}

1. Patel KR, Cherian J, Gohil K, Atkinson D. Schizophrenia: overview and treatment options. Pharm Ther 2014;39:638-45.

2. Upthegrove R, Marwaha S, Birchwood M. Depression and schizophrenia: cause, consequence or trans-diagnostic issue? Schizophram Bull 2016;22:240-4.

3. Koutsouleris N, Meisenzahl EM, Borgward S, Riecher Rossler A, Frodl T, Kambeitz J, Kohler Y, et al. Individualized differential diagnosis of schizophrenia and mood disorders using neuroanatomical biomarkers. Brain 2015;138:2059-73.

4. Mittal D, Ali A, Md S, Baboota S, Sahni JK, Ali J. Insights into the direct nose to brain delivery: current status and future perspective. Drug Delivery 2014;21:75-86.

5. Lu CT, Zhao YZ, Wong HL, Cai J, Peng L, Tian XQ. Current approaches to enhance CNS delivery of drugs across the brain barriers. Int J Nanomed 2014;9:2241-57.

6. Husean IY, Rahman Al-obaidi IRAA. Optimizing nasal for optimizing nasal formulation for the prevention of serious infections caused by simulation for prevention of serious infections caused by mrsa. Int J Pharm Pharm Sci 2014; 7:496-503.

7. Pagar SA, Shinkar DM, Saudagar RB. Development and evaluation of in situ nasal mucoadhesive gel of metoprolol succinate by using 32 full factorial design. Int J Pharm Pharm Sci 2014;6:218-23.

8. Lampel A, Ulijn RV, Tuttle T. Guiding principles for peptide nanotechnology through directed discovery. Chem Soc Rev 2018;47:3737-58.

9. Gordillo Galeano A, Mora Huertas CE. Solid lipid nanoparticles and nanostructured lipid carriers: a review emphasizing on particle structure and drug release. Eur J Pharm Biopharm 2018;133:285-308.

10. Patil TS, Deshpande AS. Nanostructured lipid carriers-based drug delivery for treating various lung diseases: a state-of-the-art review. Int J Pharm 2018;547(Suppl 2):209-25.

11. Doktorovova S, Souto EB, Silva AM. Nanotoxicology applied to solid lipid nanoparticles and nanostructured lipid carriers-a systematic review of in vitro data. Eur J Pharm Biopharm 2014;87:1-8.

12. Juven Wetzler A, Fostick L, Cwikel Hamzany S, Balaban E, Zohar J. Treatment with ziprasidone for schizophrenia patients with OCD. Eur Neuropsychopharmacol 2014;24:1454-62.

13. Devkar TB, Tekade AR, Khandelwal KR. Surface engineered nanostructured lipid carriers for an efficient nose to brain delivery of ondansetron $\mathrm{HCl}$ using delonix regia gum as a natural mucoadhesive polymer. Colloids Surf B 2014;122:14350 .

14. Singh SK, Dadhania P, Vuddanda PR, Jain A, Velaga S, Singh S. Intranasal delivery of asenapine loaded nanostructured lipid carriers: formulation, characterization, pharmacokinetics and behavioral assessment. RSC Adv 2016;6:2032-45.

15. Abdelwahab SI, Sheikh BY, Taha MM, How CW, Abdullah R, Yagoub $\mathrm{U}$, et al. Thymoquinone-loaded nanostructured lipid carriers: preparation, gastroprotection, in vitro toxicity, and pharmacokinetic properties after extravascular administration. Int J Nanomed 2013;8:2163-72.

16. Alam MI, Beg S, Samad A, Baboota S, Kohli K, Ali J, et al. Strategy for effective brain drug delivery. Eur J Pharm Sci 2010;40:385403.

17. Naseri N, Valizadeh H, Zakeri Milani P. Solid lipid nanoparticles and nanostructured lipid carriers: structure, preparation and application. Adv Pharm Bull 2015;5:305-13.

18. Shah B, Khunt D, Bhatt H, Misra M, Padh H. Intranasal delivery of venlafaxine loaded nanostructured lipid carrier: risk assessment and QbD based optimization. J Drug Delivery Sci Technol 2016;33:37-50.

19. Malik DS, Kaur G. Nanostructured gel for topical delivery of azelaic acid: Designing, characterization, and in vitro evaluation. J Drug Delivery Sci Technol 2018;47:123-36.

20. Butani S, Shah T, Parmar K, Rajput A. Development of rizatriptan benzoate microspheres for the nose to brain targeting. Int J Appl Pharm 2016;8:69-74.

21. Madane RG, Mahajan HS. Curcumin-loaded nanostructured lipid carriers (NLCs) for nasal administration: design, characterization, and in vivo study. Drug Delivery 2016;23:1326-34.

22. Galgatte UC, Kumbhar AB, Chaudhari PD. Development of in situ gel for nasal delivery: design, optimization, in vitro and in vivo evaluation. Drug Delivery 2014;21:62-73.

23. Sood S, Jain K, Gowthamarajan K. Optimization of curcumin nanoemulsion for intranasal delivery using design of experiment and its toxicity assessment. Colloids Surf B 2014;113:330-7.

24. Gartziandia O, Herran E, Pedraz JL, Carro E, Igartua M, Hernandez RM. Chitosan coated nanostructured lipid carriers for the brain delivery of proteins by intranasal administration. Colloids Surf B 2015;134:304-13.

25. Shah B, Khunt D, Misra M, Padh H. Non-invasive intranasal delivery of quetiapine fumarate loaded microemulsion for brain targeting: formulation, physicochemical and pharmacokinetic consideration. Eur J Pharm Sci 2016;91:196207.

26. Gabal YM, Kamel AO, Sammour OA, Elshafeey AH. Effect of surface charge on the brain delivery of nanostructured lipid carriers in situ gels via the nasal route. Int J Pharm 2014;473:42-57.

27. Kajal S Jadhav, Kiran B Erande. Solubility enhancement and formulation of fast dissolving tablets of ziprasidone hydrochloride. Int J Res Pharm Chem 2016;6:675-83.

28. Kaur N, Sharma K, Bedi N. Topical nanostructured lipid carrierbased hydrogel of mometasone furoate for the treatment of psoriasis. Pharm Nanotechnol 2018;6:133-43.

29. Jain K, Sood S, Gowthamarajan K. Optimization of artemetherloaded NLC for intranasal delivery using central composite design. Drug Delivery 2015;22:940-54.

30. Khan A, Imam SS, Aqil M, Ahad A, Sultana Y, Ali A, et al. Brain targeting of temozolomide via the intranasal route using lipidbased nanoparticles: brain pharmacokinetic and scintigraphic analyses. Mol Pharm 2016;13:3773-82.

31. Li F, Weng Y, Wang L, He H, Yang J, Tang X. The efficacy and safety of bufadienolides-loaded nanostructured lipid carriers. Int J Pharm 2010;393:204-12.

32. Dhuria SV, Hanson LR, Frey II WH. Intranasal delivery to the central nervous system: mechanisms and experimental considerations. J Pharm Sci 2010;99:1654-73. 